Impaired mitochondrial oxidative phosphorylation and fatty acid oxidation with enhanced mitochondrial oxidative stress in spontaneously-occurring feline hypertrophic cardiomyopathy

Yokota, Takashi; Christiansen, Liselotte Bruun; Dela, Flemming

Published in:

Journal of Cardiac Failure

DOI:

10.1016/j.cardfail.2014.07.098

Publication date:

2014

Document version

Early version, also known as pre-print

Citation for published version (APA):

Yokota, T., Christiansen, L. B., \& Dela, F. (2014). Impaired mitochondrial oxidative phosphorylation and fatty acid oxidation with enhanced mitochondrial oxidative stress in spontaneously-occurring feline hypertrophic cardiomyopathy. Journal of Cardiac Failure, 20 (10 supplement), S147. [O-008].

https://doi.org/10.1016/j.cardfail.2014.07.098 


\section{Free Communications: Oral}

\section{O-005}

A Noble Target Molecule of Nobiletin is Crucial for Cardiac Hypertrophic Responses

YOICHI SUNAGAWA ${ }^{1,2}$, MASAFUMI FUNAMOTO ${ }^{1}$, MASATOSHI FUJTA ${ }^{3}$, KOJI HASEGAWA ${ }^{2}$, TATSUYA MORIMOTO ${ }^{1}$

${ }^{1}$ Division of Molecular Medicine, School of Pharmaceutical Sciences, University of Shizuoka, Shizuoka, Japan, ${ }^{2}$ Division of Translational Research, Clinical Research Institute, Kyoto Medical Center, National Hospital Organization, Kyoto, Japan, ${ }^{3}$ Social Welfare Corporation Uji Hospital, Kyoto, Japan

Introduction: Maladaptive hypertrophy is being recognized as a critical event during the development of heart failure. We found that nobiletin, a poly-methoxy flavonoids derived from Citrus unshu, repressed hypertrophic responses in cardiomyocytes and prevented the development of heart failure after myocardial infarction in rats.

Thus, nobiletin is expected as a useful agent for heart failure. However, the target molecule of nobiletin in cardiomyocytes is still unclear. Methods and Results: First, we investigated the localization of nobiletin within cardiomyocytes by generating nobiletin conjugated with Tokyo-green, a fluorescein analogue (TG-nobiletin). We confirmed that TG-nobiletin retains its ability to inhibit cardiomyocyte hypertrophy in a manner similar with natural nobiletin, and found TG-nobiletin was distributed in a cytoplasm of cardiomyocytes. Next, to identify nobiletin-binding proteins (NBPs), we synthesized biotin-conjugated nobiletin (Bio-nobiletin). Protein extracts form rat hearts were incubated with Bio-nobiletin or Biotin alone and binding proteins were precipitated with streptavidin beads. By mass-spectrometric analysis, we identified 162 novel NBPs in the heart. In addition, Bionobiletin pull-down assay showed that Bio-nobiletin could interact with recombinant NBP1, which related to metabolic processes. In cultured cardiomyocytes, knockdown of NBP1 lost the inhibitory effects of nobiletin against phenylephrine-induced cardiomyocyte hypertrophy. Conversely, overexpression of NBP1 inhibited phenylephrine-induced hypertrophy. Conclusions: These results suggest that NBP1 may be a novel molecular target of nolitetin, a potent therapeutic agent for cardiac hypertrophy.

\section{O-007}

Mitochondrial Function as a Potential Mediator of Substrate Switching in the Heart

TAKAO KATO ${ }^{1}$, TETSUO SHIOI ${ }^{2}$, AKIRA KAWAMOTO ${ }^{2}$, TAKESHI KIMURA ${ }^{1}$ Cardiovascular Center, the Tazuke Kofukai Medical Research Institute, Kitano Hospital, ${ }^{2}$ Department of Cardiovascular Medicine, Graduate School of Medicine, Kyoto University

Purpose: We previously reported that glucose uptake increased markedly and fatty acid uptake decreased at the congestive heart failure (CHF) stage, associated with decreased gene expression related to mitochondrial function in a rat model of heart failure. The purpose of the study was to clarify the link between the changes of substrate utilization and mitochondrial function. Methods and Results: Carbonyl cyanide m-chlorophenylhydrazone (CCCP), a compound known to reduce the mitochondrial membrane potential, was injected intraperitoneally into SpragueDawley rats. The rats administered CCCP showed a transiently decreased systolic function on echocardiography and a decrease in cardiac phosphocreatine and ATP compared to the vehicle group on in vivo ${ }^{31} \mathrm{P}$ magnetic resonance spectroscopy. Since ATP was mainly produced by mitochondrial membrane potentials, decreased ATP caused by CCCP injection means dispersion of the mitochondrial function. In ex vivo perfused hearts, CCCP also decreased the mitochondrial membrane potential assessed by two-photon laser microscopy. The glucose uptake increased 1.96 fold compared to the vehicle group and the fatty acid uptake decreased 0.48 fold $90 \mathrm{mi}-$ nutes after CCCP administration, indicating that mitochondrial dysfunction caused the changes in substrate uptake. Conclusions: This rat model using CCCP could be used as a model of mitochondrial dysfunction. Mitochondrial dysfunction may be closely associated with metabolic substrat switching in the heart, especially in the failing heart.

\section{O-008}

Impaired Mitochondrial Oxidative Phosphorylation and Fatty Acid Oxidation with Enhanced Mitochondrial Oxidative Stress in Spontaneously-occurring Feline Hypertrophic Cardiomyopathy TAKASHI YOKOTA ${ }^{1}$, LISELOTTE B. CHRISTIANSEN ${ }^{2}$, FLEMMING DELA ${ }^{1}$ Department of Cardiovascular Medicine, Hokkaido University Graduate School of Medicine, Sapporo, Japan, ${ }^{2}$ Department of Biomedical Sciences, University of Copenhagen, Copenhagen, Denmark

Mitochondria play a crucial role in the development of various cardiovascular diseases. The aim of the study was to elucidate the role of mitochondrial dysfunction and oxidative stress in hypertrophic cardiomyopathy (HCM).
The cardiac and skeletal muscle were obtained from 9 spontaneously-occurring domestic HCM cats with preserved systolic function and 13 age-matched control cats. The capacities of mitochondrial oxidative phosphorylation (OXPHOS) and fatty acid oxidation were assessed with permeabilized muscle fibers and isolated mitochondria by using high-resolution respirometry. Reactive oxygen species (ROS) generation originated from isolated mitochondria was assessed by using spectrofluorometer. Thiobarbituric reactive substances (TBARS), lipid peroxides, were also measured as an oxidative stress marker. Mitochondrial state 3 respiration with complex I-linked substrates and fatty acid oxidation in the heart were significantly depressed in HCM cats. Furthermore, mitochondrial ROS generation during state 3 with complex I-linked substrates and TBARS in the heart were significantly greater in HCM cats. In contrast, there were no significant differences in mitochondrial function and oxidative stress in the skeletal muscle. Mitochondrial OXPHOS capacity with complex I-linked substrates and fatty acid oxidation were impaired with enhanced mitochondrial oxidative stress in feline HCM heart. These findings provide new insight into pathophysiology of HCM and support the hypothesis that to restore normal redox-state is beneficial in the treatment of $\mathrm{HCM}$.

\section{O-009}

Mst1 Plays a Cell-protective Role in the Heart Through FoxO1 and C/EBP- $\beta$ Phosphorylation

YASUHIRO MAEJIMA ${ }^{1}$, JUNICHI SADOSHIMA ${ }^{2}$, MITSUAKI ISOBE

${ }^{1}$ Department of Cardiovascular Medicine, Tokyo Medical and Dental University, ${ }^{2}$ Department of Cell Biology and Molecular Medicine, Rutgers New Jersey Medical School

Mst1 kinase promotes apoptosis, thereby playing a crucial role in mediating cardiac dysfunction in response to stress. Mst1 also stimulates a cell-protective mechanism through FoxO1 as negative feedback to counteract its strong pro-apoptotic actions. In order to clarify how Mst1 mediates this cell-protective mechanism in the heart, TgDN-Mst 1 were subjected to 2 -h of prolonged ischemia. Tg-DN-Mst1 hearts exhibited a significantly greater infarcted area than wild-type hearts, suggesting that the cardioprotective effect of Mst1 dominates the pro-apoptotic effect. FoxO1 upregulated antioxidant genes, including catalase, and inhibited pro-apoptotic genes, including FasL, in cardiomyocytes, and these effects upon gene expression were attenuated when endogenous Mst1 was inhibited. Chromatin immunoprecipitation assays revealed that Mst1 inhibits binding of FoxO1 to the FasL promoter, but promotes binding of FoxO1 to the catalase promoter. Reporter gene assays indicated that the C/EBP- $\beta$ binding element in the catalase promoter, but not the FoxO1 binding element, is critical for Mst1-mediated catalase gene upregulation. Interaction between FoxO1 and C/EBP- $\beta$ was enhanced in the presence of Mst1. Mass spectrometry showed that Mst 1 phosphorylates C/EBP- $\beta$ at Thr ${ }^{299}$. Phosphorylation of C/EBP- $\beta$ by Mst 1 promoted binding of C/EBP$\beta$ to DNA. Knockdown of C/EBP- $\beta$ reversed the protective effects of FoxO1 against Mst1-induced apoptosis. In summary, Mst1-mediated phosphorylation of both FoxO1 and C/EBP- $\beta$ stimulates a cell-protective mechanism by facilitating FoxO1C/EBP- $\beta$ interaction and C/EBP- $\beta$-mediated transcription in the heart.

\section{O-010}

Wnt/ $\beta$-catenin Signaling Promotes Heart Failure-Induced Skeletal Myopathy through Direct Interaction with FoxO

KATSUKI OKADA $^{1}$, ATSUHIKO NAITO $^{1,2}$, ICHIRO SHIOJIMA ${ }^{3}$, YASUSHI SAKATA $^{1}$, ISSEI KOMURO

${ }^{1}$ Department of Cardiovascular Medicine, Osaka University Graduate School of Medicine, Osaka, Japan, ${ }^{2}$ Department of Cardiovascular Medicine, Graduate School of Medicine, The University of Tokyo, Tokyo, Japan, ${ }^{3}$ Department of Medicine II, Kansai Medical University, Osaka, Japan

Heart failure-induced skeletal myopathy is a major cause of exercise intolerance in the patients of chronic heart failure (CHF). During skeletal myopathy, muscle fiber type shifts from type I fatigue resistant fiber toward type IIb fatigable fiber. Forkhead box $\mathrm{O}$ (FoxO) transcription factors have been reported to mediate skeletal myopathy in $\mathrm{CHF}$. We recently reported that serum from $\mathrm{CHF}$ model mice activated Wnt/ $\beta$-catenin signaling more potently than serum from normal mice. Recent studies have revealed that $\beta$-catenin activates FoxO signaling as well as TCF-mediated canonical Wnt signaling. Here, we investigated the molecular mechanism of skeletal myopathy during CHF. We used dilated cardiomyopathy (DCM) model mice caused by troponin mutation as $\mathrm{CHF}$ model.

Immunofluorescent staining revealed that type IIb fatigable fiber was increased in skeletal muscle of DCM mice.

Expression of both TCF and FoxO target gene was increased in the skeletal muscle of DCM model mice, which was associated with nuclear accumulation of $\beta$-catenin and FoxO1 protein. Both recombinant Wnt3a and the serum from DCM model mice promoted FoxO1 nuclear translocation, increased FoxO target gene expression, and altered the muscle fiber type toward type IIb fiber in $\mathrm{C} 2 \mathrm{C} 12$ myotubes. These results suggest that increased serum Wnt activity in CHF promotes skeletal myopathy through direct interaction with FoxO signaling. Our findings may provide new therapeutic targets of skeletal myopathy in $\mathrm{CHF}$. 